UT-892

\title{
Cosmological Gravitino Problem in Gauge-Mediated Supersymmetry Breaking Models
}

\author{
T. Asaka, K. Hamaguchi, and Koshiro Suzuki \\ Department of Physics, University of Tokyo, \\ Tokyo 113-0033, Japan
}

(May 15, 2000)

\begin{abstract}
We investigate the cosmological gravitino problem in gauge-mediated supersymmetry breaking models, where the gravitino becomes in general the lightest supersymmetric particle (LSP). In order to avoid the overclosure of the stable gravitino, the reheating temperature of inflation $T_{R}$ should be low enough. Furthermore, if the gravitino mass is larger than about $100 \mathrm{MeV}$, the decay of the next-to-LSP (NLSP) into the gravitino may modify disastrously the abundances of the light elements predicted by the big-bang nucleosynthesis (BBN). We consider the case in which the lighter stau is the NLSP and derive cosmological constraints from the BBN on the stau NLSP decay. We obtain a lower bound on the mass of stau $m_{\widetilde{\tau}_{1}}$, which is more stringent than the current experimental limit $m_{\widetilde{\tau}_{1}}>90$ $\mathrm{GeV}$ for the gravitino mass region $m_{3 / 2} \gtrsim 5 \mathrm{GeV}$. This lower bound, together with the overclosure constraint on the stable gravitino, gives an upper bound on $T_{R}$. We find that the reheating temperature can be as high as $10^{9}-10^{10} \mathrm{GeV}$ for $m_{3 / 2} \simeq 5$ $100 \mathrm{GeV}$.
\end{abstract}




\section{Introduction}

Supersymmetry (SUSY) has been considered as an attractive candidate for physics beyond the standard model. Many mechanisms have been proposed for SUSY breaking and its transmission to our sector. Among them, the gauge-mediated SUSY breaking (GMSB) models [1, 2] are fascinating since they beautifully solve the problem of flavor changing processes inherent in the SUSY standard model. Moreover, GMSB models are determined by a few parameters, and thus have high predictability. In general, they predict that the gravitino is the lightest SUSY particle (LSP) and the next-tolightest SUSY particle (NLSP) is either the lightest neutralino (almost purely bino $\widetilde{B}$ ) or the lighter stau $\widetilde{\tau}_{1}$ [3]. This mass spectrum gives striking features to the low energy phenomenology. Here we focus on the cosmological aspects of GMSB models.

The light stable gravitino gives rise to a serious cosmological problem, known as the "gravitino problem" [4, 5, 6]. The energy density of the gravitinos which are produced in the early universe may exceed the present critical density if the gravitino mass is $m_{3 / 2} \gtrsim$ $1 \mathrm{keV}$ [4]. Even if a primordial inflation is assumed, this problem cannot be solved completely since gravitinos are reproduced after the inflation ends. At the reheating epoch, gravitinos are produced by scatterings in the thermal bath. It has been pointed out in Ref. [7] that gravitinos might also be produced non-thermally at the preheating epoch. Although this non-thermal mechanism might dominate the thermal production, we will not consider it, since it highly depends on inflation models and we would like to have a general discussion.

Stable gravitinos which are produced thermally do not overclose the universe if the reheating temperature of the inflation $T_{R}$ is low enough. The upper bound on $T_{R}$ is given by $[5,6$

$$
T_{R} \lesssim\left\{\begin{array}{ll}
100 \mathrm{GeV} \text { to } 1 \mathrm{TeV} & \text { for } 1 \mathrm{keV} \lesssim m_{3 / 2} \lesssim 100 \mathrm{keV} \\
10^{8} \mathrm{GeV} \times\left(\frac{m_{3 / 2}}{1 \mathrm{GeV}}\right)\left(\frac{m_{\widetilde{B}}}{100 \mathrm{GeV}}\right)^{-2} & \text { for } m_{3 / 2} \gtrsim 100 \mathrm{keV}
\end{array},\right.
$$

where $m_{\widetilde{B}}$ denotes the bino mass. Since this upper bound on $T_{R}$ is so severe for a lighter gravitino mass region?, inflation models which predict high reheating temperatures are allowed only for a larger $m_{3 / 2}$ region.

However, when one considers a heavy gravitino (say, $m_{3 / 2} \gtrsim 100 \mathrm{MeV}$ ) in GMSB, there is another cosmological difficulty associated with the NLSP decay. The NLSP decays into the LSP gravitino only through gravitational interaction and its lifetime will

\footnotetext{
${ }^{1}$ We assume here that the $R$-parity is exact and hence the LSP gravitino is stable.

${ }^{2}$ Of course, there is no gravitino problem for $m_{3 / 2} \lesssim 1 \mathrm{keV}[$ 田.
} 
be comparable to the big-bang nucleosynthesis $(\mathrm{BBN})$ era $\left(t \sim 1-10^{2} \mathrm{sec}\right)$. The decay of the NLSP during or after the BBN is dangerous, since the decay products might alter the abundances of the light elements, which spoils the success of the BBN [8, 9, 10]. To avoid this difficulty, the lifetime and the energy density of the NLSP are severely restricted [5, 11, 12, 13.

In this letter, we investigate cosmological difficulties associated with the stable gravitino and derive the most conservative upper bound on the reheating temperature. Especially, we consider in detail the cosmological consequences of the NLSP decay into the gravitino around the BBN epoch. Similar analysis had been made in Ref. [13], in which the BBN constraint is obtained by considering mainly the effects of hadrons produced in the NLSP decay. However, the BBN with high energy hadron injection, examined in Refs. [9, 10], has not completely been settled yet, since there exist some uncertainties[. Here, since we would like to obtain the most conservative constraint on the reheating temperature, we will focus on the BBN constraint coming from the photo-dissociation of the light elements by the NLSP decay. Furthermore, we consider the case when the lighter stau $\widetilde{\tau}_{1}$ is the NLSP. This is because, compared to the $\widetilde{B}$ NLSP, the $\widetilde{\tau}_{1}$ NLSP has larger annihilation cross sections and so its abundance when it decays is smaller, which results in a weaker constraint on the reheating temperature.

\section{Estimation of $\tilde{\tau}_{1}$ NLSP abundance}

First of all, we discuss cosmological evolution of the $\widetilde{\tau}_{1}$ NLSP and estimate its abundance when it decays, since the abundance is crucial for obtaining BBN constraints. Let us start from a brief thermal history of $\widetilde{\tau}_{1}$. By the time when the cosmic temperature is comparable to the $\widetilde{\tau}_{1}$ mass $\left(T \simeq m_{\widetilde{\tau}_{1}}\right)$, only the $\widetilde{\tau}_{1}$ NLSP among the SUSY particles is in thermal equilibrium with the standard model particles. When $\widetilde{\tau}_{1}$ 's become nonrelativistic for $T \lesssim m_{\widetilde{\tau}_{1}}$, they pair-annihilate into standard model particles and the abundance decreases exponentially. At $T \simeq T_{f}\left(T_{f}\right.$ : the freeze-out temperature of $\widetilde{\tau}_{1}$ 雨), $\widetilde{\tau}_{1}$ decouples from the thermal bath and its abundance freezes out. Finally, when the Hubble parameter $H$ becomes comparable to the decay rate, $\widetilde{\tau}_{1}$ decays into the gravitino. The evolution of $\widetilde{\tau}_{1}$ described above can be traced by solving the Boltzmann equation.

Before writing down the equation, we must take care of the following two facts. The first one is that the masses of charged sleptons $\widetilde{\tau}_{1}, \widetilde{\mu}_{1}$, and $\widetilde{e}_{1}$ (the subscript 1 denotes

\footnotetext{
${ }^{3}$ For example, there exist uncertainties of the experimental data of the hadron scattering processes, and also of the statistical treatment of the errors.

${ }^{4}$ In our case, we find $T_{f} / m_{\widetilde{\tau}_{1}} \simeq 1 / 28-1 / 25$ by numerical calculation.
} 
the lighter mass eigenstates) are almost degenerate in GMSB models. This is because they are mostly right-handed, and hence receive at the messenger scale the same soft masses which are determined by the gauge quantum numbers. The mass differences between them at the weak scale are induced from the renormalization group effects due to the leptonic Yukawa couplings $y_{i}(i=e, \mu, \tau)$ and also from the left-right mixings through $y_{i}$. Considering the observed masses of charged leptons, we safely neglect $y_{e}$ and $y_{\mu}$, and hence the masses for $\widetilde{\mu}_{1}$ and $\widetilde{e}_{1}, m_{\widetilde{\mu}_{1}}$ and $m_{\widetilde{e}_{1}}$, are the same. On the other hand, the tau Yukawa coupling $y_{\tau}$ gives negative contribution to the stau mass when it is evolved from the messenger scale to the weak scale, and the left-right mixing between the staus also decreases $m_{\widetilde{\tau}_{1}}$. Both effects lead to the fact that $m_{\widetilde{\tau}_{1}}$ is always smaller than $m_{\widetilde{\mu}_{1}\left(\widetilde{e}_{1}\right)}$. This mass splitting $\Delta m \equiv m_{\widetilde{\mu}_{1}\left(\widetilde{e}_{1}\right)}-m_{\widetilde{\tau}_{1}}$ plays a crucial role in calculating the $\widetilde{\tau}_{1}$ abundance. If $\Delta m$ is very small, the relic abundance of $\widetilde{\tau}_{1}$ is determined by not only its annihilation processes, but also by the annihilation with $\widetilde{\mu}_{1}$ and $\widetilde{e}_{1}$, i.e., we should include the coannihilation effects [14]. We have verified numerically that these coannihilation effects become significant for $\Delta m \lesssim T_{f}$. In GMSB models, both $\Delta m \lesssim T_{f}$ and $\Delta m \gtrsim$ $T_{f}$ are allowed

Secondly, if the bino mass $m_{\widetilde{B}}$ is close to $m_{\widetilde{\tau}_{1}}$, we should also take into account the coannihilation of $\widetilde{\tau}_{1}$ with $\widetilde{B}$, as well as with $\widetilde{\mu}_{1}$ and $\widetilde{e}_{1}$. However, this effect increases the abundance of $\widetilde{\tau}_{1}$, which results in more stringent upper bound on $T_{R}$ (see the following discussions). Thus, we forbid the coannihilation of $\widetilde{\tau}_{1}$ with $\widetilde{B}$ and restrict ourselves in the parameter region

$$
m_{\widetilde{B}}>m_{\widetilde{\tau}_{1}}+T_{f}
$$

In the actual calculation below, we take the lower bound on $m_{\widetilde{B}}$ as $m_{\widetilde{B}} \geq 1.1 m_{\widetilde{\tau}_{1}}$ to be conservative.

Now we are at the point to present the Boltzmann equation including the coannihilation effects. This equation describes the evolution of the total number density of charged sleptons $n=n_{\widetilde{\tau}_{1}}+n_{\widetilde{\tau}_{1}^{*}}+n_{\widetilde{\mu}_{1}}+n_{\widetilde{\mu}_{1}^{*}}+n_{\widetilde{e}_{1}}+n_{\widetilde{e}_{1}^{*}}$, where $n_{i}$ stands for the number density of one slepton species 诃 [14,

$$
\frac{d n}{d t}=-3 H n-\left\langle\sigma_{\text {eff }} v\right\rangle\left[n^{2}-\left(n^{\mathrm{eq}}\right)^{2}\right]
$$

where

$$
\left\langle\sigma_{\mathrm{eff}} v\right\rangle=\sum_{i, j}\left\langle\sigma_{i j} v\right\rangle \frac{n_{i}^{\mathrm{eq}}}{n^{\mathrm{eq}}} \frac{n_{j}^{\mathrm{eq}}}{n^{\mathrm{eq}}} .
$$

\footnotetext{
${ }^{5}$ The mass difference $\Delta m$ becomes larger as $\tan \beta$ increases, where $\tan \beta \equiv v_{u} / v_{d}$ ( $v_{u}$ and $v_{d}$ denote the vacuum expectation values of Higgs fields which couple to up-type and down-type quarks, respectively).

${ }^{6}$ We distinguish particles from their anti-particles.
} 
Here $n^{\text {eq }}\left(n_{i}^{\text {eq }}\right)$ is the equilibrium value of $n\left(n_{i}\right)$ and $v$ is the relative velocity of particles $i$ and $j$. The bracket denotes the thermal average and $\sigma_{i j}$ is the total annihilation cross section of $i+j \rightarrow X+X^{\prime}$ :

$$
\sigma_{i j}=\sum_{X, X^{\prime}} \sigma\left(i+j \rightarrow X+X^{\prime}\right)
$$

where $X$ and $X^{\prime}$ represent the possible standard model particles. Note that the Boltzmann equation (3) also holds for the case where the coannihilations of $\widetilde{\tau}_{1}$ with $\widetilde{\mu}_{1}$ and $\widetilde{e}_{1}$ do not occur.

Next we discuss the thermal-averaged cross sections $\left\langle\sigma_{\text {eff }} v\right\rangle$ in Eq. (3). The thermalaveraged cross sections $\left\langle\sigma_{i j} v\right\rangle$ in Eq. (蛋 can be expanded in terms of $T / m_{\widetilde{\tau}_{1}}$ [see Eqs. (可) - (8) below]. Since the final abundance is determined by $\left\langle\sigma_{\text {eff }} v\right\rangle$ at $T \sim T_{f}$ and the freeze out temperature is typically $T_{f} \simeq m_{\widetilde{\tau}_{1}} / 25$, it is sufficient only to consider the leading term, i.e., the $s$-wave cross sections of the sleptons. Note that the $s$-wave component of the thermal-averaged cross section $\left\langle\sigma_{i j} v\right\rangle$ is equal to that of $\sigma_{i j}$. The relevant (co)annihilation channels are

$$
\begin{aligned}
& \text { (I) }\left\{\begin{array}{l}
\widetilde{\tau}_{1} \widetilde{\tau}_{1}^{*} \\
\widetilde{\mu}_{1} \widetilde{\mu}_{1}^{*} \\
\widetilde{e}_{1} \widetilde{e}_{1}^{*}
\end{array} \rightarrow \gamma \gamma, Z \gamma, Z Z, W^{+} W^{-}, f \bar{f}, h^{0} h^{0}\right. \\
& (\mathrm{II}) \quad\left\{\begin{array}{l}
\widetilde{\tau}_{1} \widetilde{\tau}_{1} \rightarrow \tau \tau \\
\widetilde{\tau}_{1}^{*} \widetilde{\tau}_{1}^{*} \rightarrow \bar{\tau} \bar{\tau} \\
\widetilde{\mu}_{1} \widetilde{\mu}_{1} \rightarrow \mu \mu \\
\widetilde{\mu}_{1}^{*} \widetilde{\mu}_{1}^{*} \rightarrow \bar{\mu} \bar{\mu} \\
\widetilde{e}_{1} \widetilde{e}_{1} \rightarrow e e \\
\widetilde{e}_{1}^{*} \widetilde{e}_{1}^{*} \rightarrow \bar{e} \bar{e}
\end{array}\right. \\
& (\mathrm{III}) \quad\left\{\begin{array}{l}
\widetilde{\tau}_{1} \widetilde{\mu}_{1} \rightarrow \tau \mu \\
\widetilde{\tau}_{1}^{*} \widetilde{\mu}_{1}^{*} \rightarrow \bar{\tau} \bar{\mu} \\
\widetilde{\tau}_{1} \widetilde{e}_{1} \rightarrow \tau e \\
\widetilde{\tau}_{1}^{*} \widetilde{e}_{1}^{*} \rightarrow \bar{\tau} \bar{e} \\
\widetilde{\mu}_{1} \widetilde{e}_{1} \rightarrow \mu e \\
\widetilde{\mu}_{1}^{*} \widetilde{e}_{1}^{*} \rightarrow \bar{\mu} \bar{e}
\end{array}\right. \\
& (\mathrm{IV})\left\{\begin{array}{l}
\widetilde{\tau}_{1} \widetilde{\mu}_{1}^{*} \rightarrow \tau \bar{\mu}, \nu_{\tau} \bar{\nu}_{\mu} \\
\widetilde{\tau}_{1}^{*} \widetilde{\mu}_{1} \rightarrow \bar{\tau} \mu, \bar{\nu}_{\tau} \nu_{\mu} \\
\widetilde{\tau}_{1} \widetilde{e}_{1}^{*} \rightarrow \tau \bar{e}, \nu_{\tau} \bar{\nu}_{e} \\
\widetilde{\tau}_{1}^{*} \widetilde{e}_{1} \rightarrow \bar{\tau} e, \bar{\nu}_{\tau} \nu_{e} \\
\widetilde{\mu}_{1} \widetilde{e}_{1}^{*} \rightarrow \mu \bar{e}, \nu_{\mu} \bar{\nu}_{e} \\
\widetilde{\mu}_{1}^{*} \widetilde{e}_{1} \rightarrow \bar{\mu} e, \bar{\nu}_{\mu} \nu_{e}
\end{array}\right.
\end{aligned}
$$

Here $f$ denotes the ordinary quarks and leptons. 
We calculate cross sections for all the processes listed in (I)-(IV). The processes (I) and (II) represent the annihilation of each slepton species, while (III) and (IV) describe coannihilation processes. In the following we briefly explain their features. First of all, let us estimate the cross sections for processes (I). Although they have many final states, it turns out that most of them give very small contributions to the total cross section. Processes which are induced by the tau Yukawa coupling and/or the left-right mixing of sleptons are suppressed due to their smallness[]. Furthermore, in calculating $s$-wave cross sections, derivative couplings of the sleptons vanish. From these considerations, we find that $\gamma \gamma$ and $Z \gamma$ are the dominant channels 8 , and $\left\langle\sigma_{\widetilde{\tau}_{1}} \widetilde{\tau}_{1}^{*} v\right\rangle$ is approximately given by

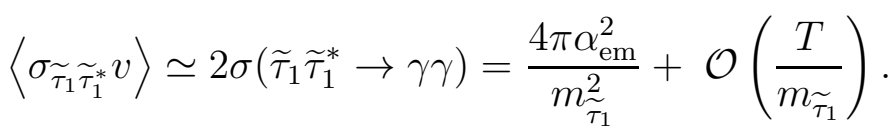

Next, we turn to the processes (II), which have only $t$-channel and $u$-channel diagrams. Diagrams induced by the exchange of Higgsino and neutral Wino can be safely neglected due to the smallness of $y_{\tau}$ and left-right mixing, respectively. Then, there is only $\widetilde{B}$ contribution and its cross section is calculated as

$$
\left\langle\sigma_{\widetilde{\tau}_{1} \widetilde{\tau}_{1}} v\right\rangle \simeq \frac{16 \pi \alpha_{\mathrm{em}}^{2} m_{\widetilde{B}}^{2}}{\cos ^{4} \theta_{W}\left(m_{\widetilde{\tau}_{1}}^{2}+m_{\widetilde{B}}^{2}\right)^{2}}+\mathcal{O}\left(\frac{T}{m_{\widetilde{\tau}_{1}}}\right) .
$$

The annihilation channels listed in (III) can be calculated in the same way as

$$
\left\langle\sigma_{\widetilde{\tau}_{1} \tilde{\mu}_{1}} v\right\rangle \simeq \frac{8 \pi \alpha_{\mathrm{em}}^{2} m_{\widetilde{B}}^{2}}{\cos ^{4} \theta_{W}\left(m_{\widetilde{\tau}_{1}}^{2}+m_{\widetilde{B}}^{2}\right)^{2}}+\mathcal{O}\left(\frac{T}{m_{\tau_{1}}}\right),
$$

for $m_{\widetilde{\mu}_{1}}=m_{\widetilde{\tau}_{1}}$. Cross sections for the processes (IV) are also dominated by the $\widetilde{B}$ exchange. However, the left-right mixings of sleptons are necessary for these processes, and so they give only tiny contributions to the total thermal-averaged cross section $\left\langle\sigma_{\text {eff }} v\right\rangle$. Cross sections for other processes in (I)-(IV) are calculated similarly. From

\footnotetext{
${ }^{7}$ The mixing angles $\alpha_{i}$ of sleptons are in general small in GMSB models $\left(\sin \alpha_{i} \ll 1\right)$. However, in the extremely large $\tan \beta$ region, the stau mixing angle $\alpha_{\tilde{\tau}}$ can be large as $\sin \alpha_{\tilde{\tau}} \simeq \mathcal{O}(0.1)$. Although $\left\langle\sigma_{\widetilde{\tau}_{1} \widetilde{\tau}_{1}^{*}} v\right\rangle / \sigma\left(\widetilde{\tau}_{1} \widetilde{\tau}_{1}^{*} \rightarrow \gamma \gamma\right)$ might become of $\mathcal{O}(10)$ as opposed to Eq. (6) in this case, we find that the final upper bound on $T_{R}$ only increases by less than a factor of 2 .

8 The ratios of the cross sections for the dominant processes $\gamma \gamma, Z \gamma$, and $Z Z$ are 1.0:0.6:(0.1-0.2). The contributions of the other channels are less than a few percents.

${ }^{9}$ Actually, if $m_{\widetilde{\tau}_{1}}<m_{Z} / 2$, the $Z \gamma$ channel is forbidden kinematically. Furthermore, if $m_{\widetilde{\tau}_{1}} \simeq m_{h^{0}} / 2$ $\left(m_{h^{0}}\right.$ : mass of the lightest Higgs boson $\left.h^{0}\right)$, the annihilation cross section is enhanced by the pole contribution of $h^{0}$. These effects may change the value of $Y$ in Fig. 1. However, we simply assume Eq. (6), since the mass region of $m_{\widetilde{\tau}_{1}}<90 \mathrm{GeV}$ is excluded from the current experimental limit.
} 


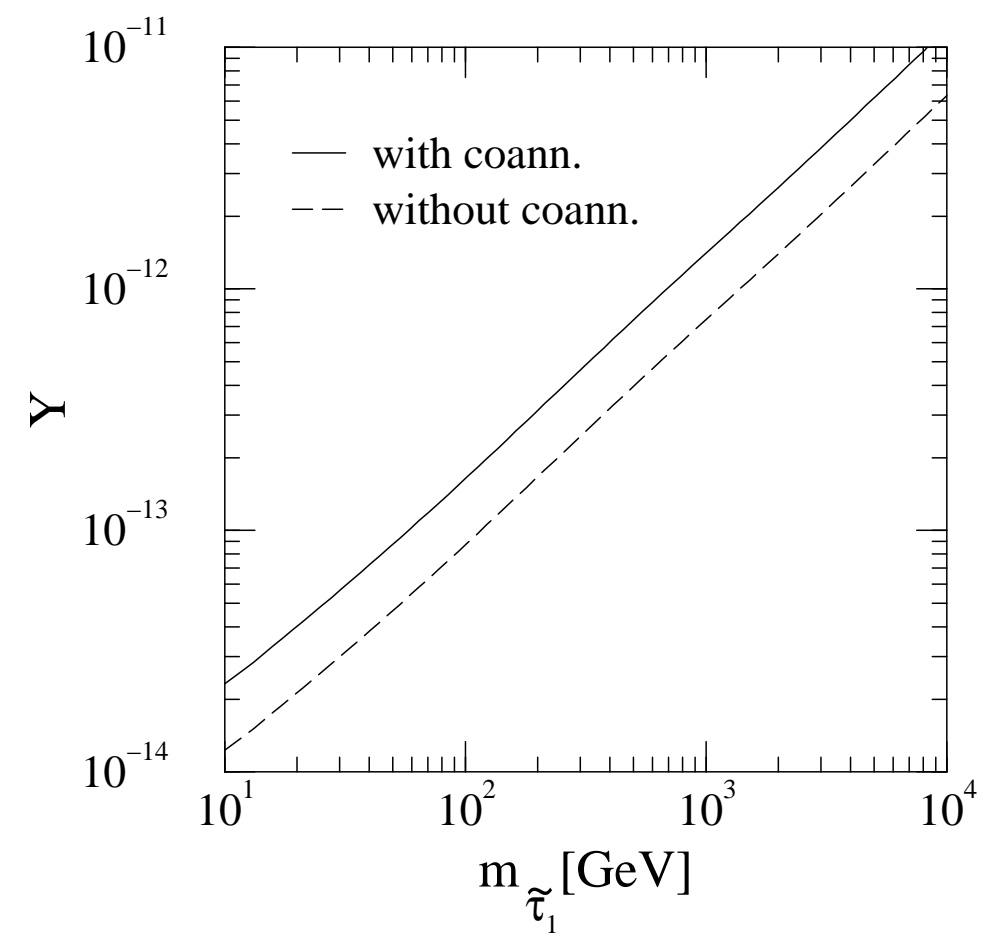

Figure 1: The final $\widetilde{\tau}_{1}$ abundance $Y$. The solid line represents the abundance for the case with coannihilation and the dashed line represents that for the case without coannihilation.

the above arguments, we conclude that all the annihilation cross sections are almost determined by only the masses of the bino and the sleptons.

Finally, including all the cross sections described above, we solve the Boltzmann equation (3) numerically. Here we take $m_{\widetilde{B}}=1.1 m_{\widetilde{\tau}_{1}}$ [see Eq. (2)]. In this case the (co)annihilation cross sections take their maximal values, which gives the smallest $\widetilde{\tau}_{1}$ abundance. In Fig. [ 1 the result is shown in terms of $Y$, which is defined by $Y \equiv n / s=$ $\sum_{i} n_{i} / s$ with the total entropy density of the universe $s$. Here are some comments. We calculate both of the cases with and without coannihilation effects as mentioned before. If the mass difference between $\widetilde{\mu}_{1}\left(\widetilde{e}_{1}\right)$ and $\widetilde{\tau}_{1}$ is large enough $\left(\Delta m \gtrsim T_{f}\right), \widetilde{\mu}_{1}$ and $\widetilde{e}_{1}$ decay into $\widetilde{\tau}_{1}$ and disappear from the thermal bath before $\widetilde{\tau}_{1}$ freezes out. Therefore, the coannihilation processes become ineffective. In this case, the abundance becomes $Y \simeq\left(n_{\widetilde{\tau}_{1}}+n_{\widetilde{\tau}_{1}^{*}}\right) / s$ for $T \lesssim T_{f}$, since $n_{\widetilde{\mu}_{1}} \simeq n_{\widetilde{e}_{1}} \simeq 0$. For $T \lesssim T_{f}$ the number density of $\widetilde{\tau}_{1}$ and the entropy density decrease at the same rate as the universe expands, and $Y$ takes a constant value until $\widetilde{\tau}_{1}$ decays. In fact, Fig. 1 shows the value of $Y$ just before the decay of $\widetilde{\tau}_{1}$. On the other hand, when $\Delta m \lesssim T_{f}, \widetilde{\mu}_{1}$ and $\widetilde{e}_{1}$ are still in thermal 
equilibrium at $T \simeq T_{f}$ due to the effect of inverse decays, and thus we should consider the coannihilations. Note that $Y$ takes a constant value after sleptons decouple from the thermal bath even in this case. This is because $Y$ is an invariant parameter against the expansion of the universe and also because $n=n_{\widetilde{\tau}_{1}}+n_{\widetilde{\tau}_{1}^{*}}+n_{\widetilde{\mu}_{1}}+n_{\widetilde{\mu}_{1}^{*}}+n_{\widetilde{e}_{1}}+n_{\widetilde{e}_{1}^{*}}$ does not change by the decays of $\widetilde{\mu}_{1}$ and $\widetilde{e}_{1}$. Therefore, the final abundance for $\widetilde{\tau}_{1}$ is given by $Y$ for both cases with and without coannihilation effects.

As shown in Fig. 1, the final abundance with coannihilation is larger than that without coannihilation. This can be understood as follows: Imagine when the coannihilation cross sections in (III) and (IV) are extremely large. In this case, the final abundance $Y$ would be smaller than that without coannihilation. On the other hand, if the coannihilation cross sections are zero, $Y$ increase by a factor of 3 because the relevant degrees of freedom is now 6 , not 2 . In our case, coannihilation cross sections in (III) and (IV) are smaller than those in (I) and (II), and the final abundance with coannihilation is about twice as large as that without coannihilation.

We present in Fig. 1 the results for the two extreme cases $\Delta m \simeq 0$ and $\Delta m \gg T_{f}$, for illustration and for comparison. In fact, we find that the final abundance of stau for the case $0 \lesssim \Delta m \lesssim T_{f}$ falls between the two lines in Fig. 1.

Before closing this section, we should comment on the case in which the mass difference between $\widetilde{\tau}_{1}$ and $\widetilde{\mu}_{1}\left(\widetilde{e}_{1}\right)$ is extremely small. If the mass difference is smaller than the tau mass (of course we should include the coannihilation effects in this case), the decay channel $\widetilde{\mu}_{1}\left(\widetilde{e}_{1}\right) \rightarrow \widetilde{\tau}_{1} \bar{\tau} \mu(e)$ is kinematically forbidden. Then, $\widetilde{\mu}_{1}$ and $\widetilde{e}_{1}$ may dominantly decay into gravitinos एand so we should consider the effect of the $\widetilde{\mu}_{1}\left(\widetilde{e}_{1}\right)$ decay on the BBN, as well as $\widetilde{\tau}_{1}$. However, we simply neglect this possibility, since the final results do not change much.

\section{$3 \quad$ BBN constraint on $\tilde{\tau}_{1}$ NLSP decay}

We are now ready to investigate the cosmological consequence of the decay of the $\widetilde{\tau}_{1}$ NLSP into the gravitino at the BBN epoch. The $\widetilde{\tau}_{1}$ decay rate is estimated, for $m_{\widetilde{\tau}_{1}} \gg m_{3 / 2}$, as

$$
\Gamma_{\widetilde{\tau}_{1}} \simeq \frac{1}{48 \pi} \frac{m_{\tau_{1}}^{5}}{m_{3 / 2}^{2} M_{*}^{2}}
$$

${ }^{10}$ The left-right mixing allows $\widetilde{\mu}_{1}$ to decay through $\widetilde{\mu}_{1} \rightarrow \nu_{\mu} \widetilde{\tau}_{1} \bar{\nu}_{\tau}$. If this is the main decay channel, the following discussion does not change, since $\widetilde{\mu}_{1}$ has already decayed into $\widetilde{\tau}_{1}$ before the $\widetilde{\tau}_{1}$ decays. 


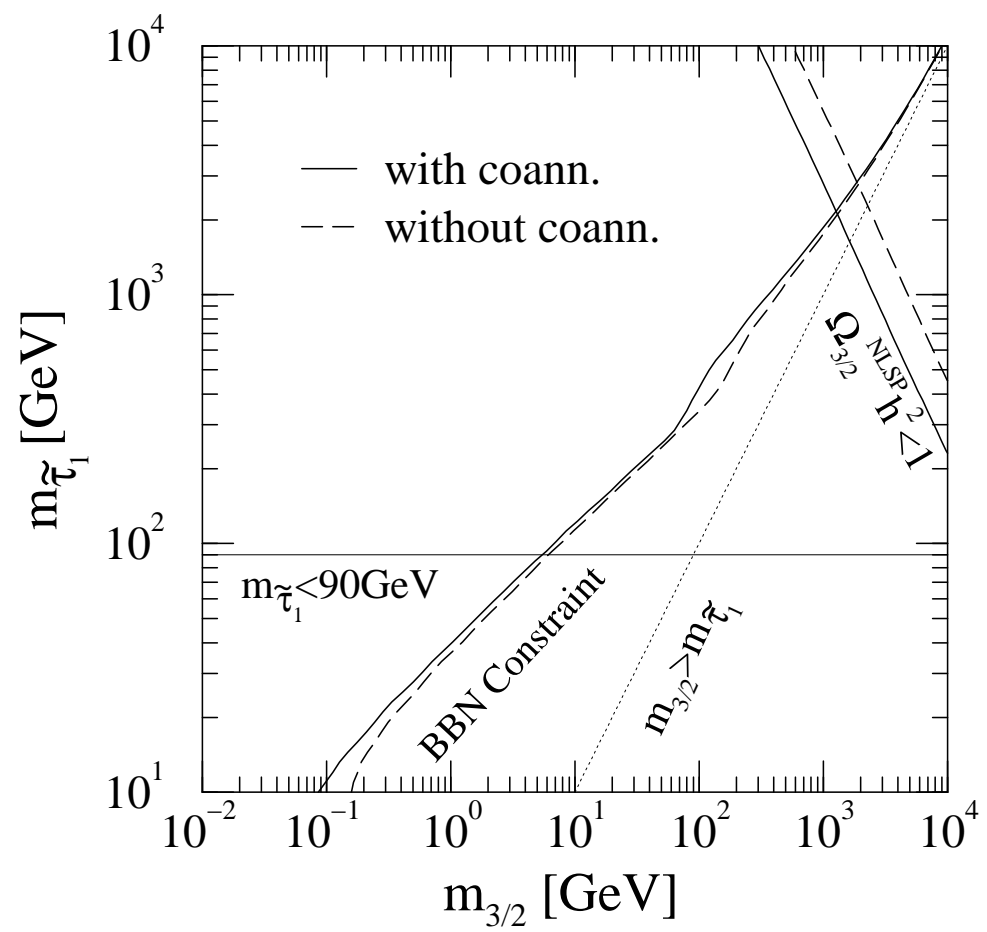

Figure 2: Various bounds on the $\widetilde{\tau}_{1}$ mass. The lower bounds on $m_{\widetilde{\tau}_{1}}$ from the BBN photo-dissociation effect are shown by the thick solid and dashed lines for the cases with and without the coannihilation effects, respectively. The experimental lower bound on $m_{\widetilde{\tau}_{1}}$ is shown by thin solid line. We also present the upper bounds on $m_{\widetilde{\tau}_{1}}$ from the constraint $\Omega_{3 / 2}^{\mathrm{NLSP}} h^{2}<1$ by the thick solid and dashed lines for the cases with and without the coannihilation effects, respectively.

with $M_{*}=2.4 \times 10^{18} \mathrm{GeV}$, and the lifetime is given by

$$
\tau_{\widetilde{\tau}_{1}} \simeq 6 \times 10^{4} \sec \left(\frac{m_{3 / 2}}{1 \mathrm{GeV}}\right)^{2}\left(\frac{m_{\widetilde{\tau}_{1}}}{100 \mathrm{GeV}}\right)^{-5} .
$$

Therefore, $\widetilde{\tau}_{1}$ is found to be a long-lived particle and to decay during or even after the BBN epoch when $m_{3 / 2} \gtrsim 10 \mathrm{MeV}$ for $m_{\tau_{1}}=100 \mathrm{GeV}$, and hence we should seriously consider the effects of its decay on the BBN.

The energetic $\tau$ 's produced by the $\widetilde{\tau}_{1}$ decay trigger the electro-magnetic (EM) cascade processes and induce high energy photons. These photons may be abundant enough to destroy or overproduce various light elements (D, ${ }^{3} \mathrm{He},{ }^{4} \mathrm{He}$, etc.) synthesized by the BBN. In order to keep the success of the BBN, the energy density (per the entropy density) of the extra EM particles emitted after the BBN era (i.e., $t \gtrsim 10^{4} \mathrm{sec}$ ) is severely constrained [8]. Here it should be noted that not all the energy of tau contribute to these photo-dissociation processes. This is because the tau decays before it causes the EM 
cascade processes and the produced neutrinos give no effects on the photo-dissociation processes 巴. By using the result of the recent analysis in Ref. [12] (see Fig.16 in Ref. [12]), we can obtain an upper bound on

$$
\frac{\rho_{\tau_{1}}}{s}=m_{\tau_{1}} Y
$$

for a given lifetime of $\widetilde{\tau}_{1}$. Here $\rho_{\tau_{1}}$ denotes the energy density of $\widetilde{\tau}_{1}$. When one fixes the gravitino mass, this bound is translated into the lower bound on $m_{\tau_{1}}$, since both the

abundance and the lifetime of $\widetilde{\tau}_{1}$ are determined by its mass $m_{\widetilde{\tau}_{1}}$. The obtained result is found in Fig. 2.

You can see that this BBN photo-dissociation constraint gives a more stringent lower bound on $m_{\tau_{1}}$ for $m_{3 / 2} \gtrsim 5 \mathrm{GeV}$, compared to the experimental bound $m_{\widetilde{\tau}_{1}}>90 \mathrm{GeV}$. This result will help us to estimate the upper bound on the reheating temperature in the next section.

\section{Gravitino problem and constraint on $T_{R}$}

Finally, we discuss the cosmological gravitino problem and obtain an upper bound on the reheating temperature of inflation. At the reheating epoch after the inflation ends, gravitinos are produced thermally by scatterings with particles in the hot plasma of the universe ${ }^{\mathrm{T2}}$. The relic abundance of the gravitino is given by [5, 6]

$$
\Omega_{3 / 2}^{\mathrm{th}} h^{2} \simeq 0.3\left(\frac{m_{3 / 2}}{1 \mathrm{GeV}}\right)^{-1}\left(\frac{m_{\widetilde{B}}}{100 \mathrm{GeV}}\right)^{2}\left(\frac{T_{R}}{10^{8} \mathrm{GeV}}\right)
$$

where $h$ is the present Hubble parameter in unit of $100 \mathrm{~km} / \mathrm{sec} / \mathrm{Mpc}$, and $\Omega_{3 / 2}^{\mathrm{th}}=\rho_{3 / 2}^{\mathrm{th}} / \rho_{c}$ ( $\rho_{3 / 2}^{\text {th }}$ is the present energy density of the thermally produced gravitinos and $\rho_{c}$ is the critical density of the present universe). It is found from Eq. (12) that the overclosure limit of $\Omega_{3 / 2}^{\text {th }}<1$ puts an upper bound on $T_{R}$ as shown in Eq. (11). Notice that this upper bound on $T_{R}$, if one fixes the $\widetilde{B}$ mass, becomes more severe for the lighter gravitino mass region, and the highest reheating temperature allowed in GMSB models is achieved for a relatively heavy gravitino mass.

Furthermore, it should be noted that gravitinos are also produced by the $\widetilde{\tau}_{1}$ NLSP decays. Because one gravitino is produced per a $\widetilde{\tau}_{1}$ decay, we find that

$$
\Omega_{3 / 2}^{\mathrm{NLSP}} \equiv \frac{\rho_{3 / 2}^{\mathrm{NLSP}}}{\rho_{c}}=\frac{m_{3 / 2} Y}{\rho_{c} / s_{0}},
$$

\footnotetext{
${ }^{11}$ In fact, the high energy neutrinos produced by the tau decay also causes the EM cascade processes by scattering with the background neutrinos. However, this effect is less significant [15].

12 As mentioned in Sec. 1, we discard the non-thermal production of gravitinos at the preheating epoch in order to obtain the most conservative result.
} 


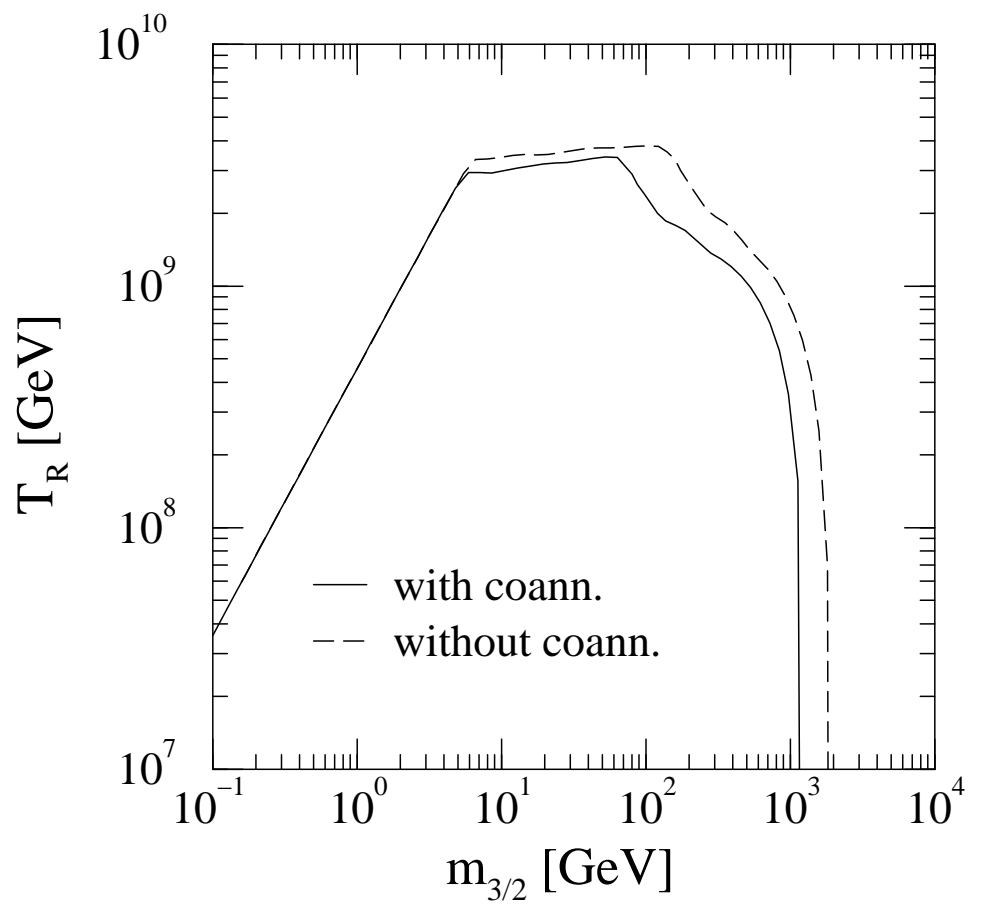

Figure 3: The upper bounds on $T_{R}$. The bounds are shown by the thick solid and dashed lines for the cases with and without the coannihilation effects, respectively.

where $\rho_{3 / 2}^{\mathrm{NLSP}}$ is the present energy density of the gravitinos produced by the $\widetilde{\tau}_{1}$ decays, and $s_{0}$ denotes the entropy density of the present universe. In Fig. 2 we also show the upper bound on $m_{\widetilde{\tau}_{1}}$ from the constraint $\Omega_{3 / 2}^{\mathrm{NLSP}} h^{2}<1$. It is found that the gravitino mass is bounded from above as $m_{3 / 2} \lesssim 1 \mathrm{TeV}$ when combined with the lower bound on $m_{\widetilde{\tau}_{1}}$ from the BBN photo-dissociation effects.

Therefore, the total relic abundance of gravitinos in GMSB models is now given by $\Omega_{3 / 2}^{\text {tot }}=\Omega_{3 / 2}^{\text {th }}+\Omega_{3 / 2}^{\text {NLSP }}$, as long as the decay of the $\widetilde{\tau}_{1}$ NLSP takes place within the age of the universe. From the lower bound on the $\widetilde{\tau}_{1}$ mass obtained in the previous section, we can estimate the most conservative upper bound on the reheating temperature in the following way. Since the abundance $\Omega_{3 / 2}^{\text {th }}$ depends on the bino mass $m_{\widetilde{B}}$ as shown in Eq. (12), the weakest bound on $T_{R}$ is obtained by the lowest value of $m_{\widetilde{B}}$. On the other hand, the lower bound on the mass of the $\widetilde{\tau}_{1}$ NLSP represented in Fig. 2 is nothing but the lower bound on $m_{\widetilde{B}}$. Now we are working in the parameter region of $m_{\widetilde{B}}$ in Eq. (目) in order to forbid the coannihilation of $\widetilde{\tau}_{1}$ with $\widetilde{B}$. Therefore, the lowest value of $m_{\widetilde{B}}$ coming from the $m_{\widetilde{\tau}_{1}}$ constraint gives the most conservative upper bound on the reheating 
temperature ${ }^{\text {[3 }}$. The result is found in Fig. 3, where we take $h=1$ for simplicity 4 .

Note that the gravitino mass region of $m_{3 / 2} \gtrsim 1 \mathrm{TeV}$ is excluded, since the gravitinos produced by the decay of $\widetilde{\tau}_{1}$ overclose the present universe $\left(\Omega_{3 / 2}^{\mathrm{NLSP}}>1\right)$. On the other hand, $\Omega_{3 / 2}^{\text {NLSP }}$ plays no role in putting an upper bound on $T_{R}$ for a lighter gravitino mass region of $m_{3 / 2} \lesssim 500 \mathrm{GeV}$.

You can see that the upper bound on $T_{R}$ is almost proportional to $m_{3 / 2}$ for $m_{3 / 2} \lesssim$ $5 \mathrm{GeV}$. In this gravitino mass region, the experimental bound on $m_{\widetilde{\tau}_{1}}$ gives the lowest value of $m_{\widetilde{B}}$ (see Fig. 2). The upper bound on $T_{R}$, thus, is the conventional one Eq.(1) with $m_{\widetilde{B}} \simeq 100 \mathrm{GeV}$. However, when $m_{3 / 2} \simeq 5 \mathrm{GeV}-100 \mathrm{GeV}$, the upper bound on $T_{R}$ takes an almost constant value of $10^{9}-10^{10} \mathrm{GeV}$. In this region the bino mass which gives the highest value of $T_{R}$ is determined by the lower bound on $m_{\widetilde{\tau}_{1}}$ from the BBN photo-dissociation constraint. Since this lower bound on $m_{\widetilde{\tau}_{1}}$ is more stringent than the experimental limit, the upper bound on $T_{R}$ also becomes more stringent than the conventional one. From Fig.2, the lower bound on the stau mass, i.e., the lower bound on the bino mass is almost proportional to $\left(m_{3 / 2}\right)^{1 / 2}$ for $m_{3 / 2}=5-100 \mathrm{GeV}$. Thus, it is found from Eq.(12) that the upper bound on $T_{R}$ becomes almost constant. Therefore, in GMSB models, the reheating temperature can be taken as high as $T_{R} \simeq 10^{9}-10^{10} \mathrm{GeV}$ for $m_{3 / 2} \simeq 5-100 \mathrm{GeV}$.

\section{Conclusions and discussions}

In this letter, we have considered the cosmological gravitino problem in GMSB models with the $\widetilde{\tau}_{1}$ NLSP. Especially, we have investigated in detail the cosmological consequence of the $\widetilde{\tau}_{1}$ decay soon after the BBN epoch. Since the $\widetilde{\tau}_{1}$ abundance when it decays is crucial for this discussion, we have solved numerically the Boltzmann equations for both cases with and without coannihilation effects, and obtained the final $\widetilde{\tau}_{1}$ abundance. We have found that the BBN constraint on the $\widetilde{\tau}_{1}$ abundance is translated into the lower bound on $m_{\widetilde{\tau}_{1}}$, and that the obtained bound is more stringent for $m_{3 / 2} \gtrsim 5 \mathrm{GeV}$ than the current experimental limit $m_{\widetilde{\tau}_{1}}>90 \mathrm{GeV}$. This gives some hints for GMSB models. If $\widetilde{\tau}_{1}$ was detected at future collider experiments, the observed $\widetilde{\tau}_{1}$ mass would enable us to set an upper bound on the gravitino mass, and hence on the SUSY breaking scale. It has also been found that the gravitino mass region of $m_{3 / 2} \gtrsim 1 \mathrm{TeV}$ is excluded (although it might be marginal considering the SUSY flavor problem), since the energy density of gravitinos produced by the decays of $\widetilde{\tau}_{1}$ 's exceeds the present critical density.

\footnotetext{
${ }^{13}$ In fact, such a value of $m_{\widetilde{B}}$ makes the $\widetilde{\tau}_{1}$ abundance smallest. (See discussions in Sec. 2.)
}

14 The upper bound on $T_{R}$ becomes more stringent for a smaller $h$. 
By using the lower bound on $m_{\widetilde{\tau}_{1}}$, we have obtained an upper bound on the reheating temperature $T_{R}$ of inflation in order to avoid the overclosure problem of the LSP gravitino produced in thermal scatterings and also in the $\widetilde{\tau}_{1}$ decay. What we have found is that the most conservative upper bound on $T_{R}$ in GMSB models is $T_{R} \lesssim 10^{9}-10^{10} \mathrm{GeV}$ when $m_{3 / 2} \simeq 5-100 \mathrm{GeV}$. This upper bound on $T_{R}$ is weaker than those in the conventional hidden sector SUSY breaking models to solve the cosmological problem of unstable gravitinos $\left(T_{R} \lesssim 10^{6} \mathrm{GeV}\right.$ and $10^{8} \mathrm{GeV}$ for $m_{3 / 2} \simeq 100-500 \mathrm{GeV}$ and $500 \mathrm{GeV}-1 \mathrm{TeV}$, respectively [12]). Therefore, it helps us a lot to build SUSY inflation models without the cosmological gravitino problem.

Such a high reheating temperature is also promising for baryogenesis. The relevant example is the leptogenesis [16] via decays of heavy Majorana neutrinos, which is very attractive from the viewpoint of the observed tiny neutrino masses. The leptogenesis requires a high reheating temperature to generate the observed baryon asymmetry of the universe四. Thus, the upper bound on the reheating temperature $T_{R} \lesssim 10^{9}-10^{10} \mathrm{GeV}$ obtained in this letter ensures some scenarios of the leptogenesis to work without the gravitino problem.

The results we have found here are almost independent on models of the GMSB, since the abundance of the $\widetilde{\tau}_{1}$ NLSP is fixed by the $\widetilde{\tau}_{1}$ mass. However, there are some loopholes in which these constraints can be avoided. If one introduces the $R$-parity breaking, the NLSP can decay before the BBN (i.e., $t \lesssim 1 \mathrm{sec}$ ) and evade the BBN constraints, and also the LSP gravitino can decay within the age of the universe. Furthermore, if one assumes the late-time entropy production such as the thermal inflation [19] in the thermal history of the universe, the abundances of the NLSP and also the LSP gravitino are diluted away so that we are free from these constraints.

In the present analysis, we have considered only the photo-dissociation effects of the decay of $\widetilde{\tau}_{1}$ on the BBN to make a conservative analysis. As pointed out in Ref. 113 the high energy hadrons produced by the $\widetilde{\tau}_{1}$ decay might also be dangerous. Here we would like to briefly comment on their effects. The high energy hadrons, if they are produced at the time $t \sim 1-10^{4} \mathrm{sec}$, delay the freeze-out of the $p-n$ conversion and raise the number ratio of $n$ to $p$, which leads to the overproduction of $\mathrm{D}$ and ${ }^{4} \mathrm{He}$ [10]. Furthermore, if $\widetilde{\tau}_{1}$ decays at the time $t \gtrsim 10^{4} \mathrm{sec}$, the produced hadrons destroy the light elements synthesized by the BBN and modify their abundances (e.g., increases the ${ }^{7} \mathrm{Li}$ abundance) [9].

\footnotetext{
${ }^{15}$ In the leptogenesis scenarios where heavy Majorana neutrinos are thermally produced, the reheating temperature of $T_{R} \gtrsim 10^{10} \mathrm{GeV}$ is required to induce the sufficient baryon asymmetry $[17]$. On the other hand, for the case when heavy Majorana neutrinos are non-thermally produced in the inflaton decays, the reheating temperature of $T_{R} \gtrsim 10^{6} \mathrm{GeV}$ is required 18 .
} 
These effects give the upper bound on the $\widetilde{\tau}_{1}$ abundance. However, there are some uncertainties in the BBN with high energy hadron injection. The relevant hadron scattering cross sections, especially those of Li, have not been observed experimentally in detail. Furthermore, the statistical treatment for the estimation of the errors has not completely settled yet. Therefore, in the present analysis we do not include the BBN constraints from the hadron injection.

\section{Acknowledgements}

We would like to thank T. Yanagida for various suggestions and stimulating discussions and also K. Kohri and Y. Nomura for useful comments. This work was partially supported by the Japan Society for the Promotion of Science (T.A. and K.H.). 


\section{References}

[1] M. Dine, A.E. Nelson, Y. Shirman, Phys. Rev. D51 (1995) 1362; M. Dine, A.E. Nelson, Y. Nir, Y. Shirman, Phys. Rev. D53 (1996) 2658.

[2] For a review, see G.F. Giudice, R. Rattazzi, Phys. Rep. 322 (1999) 419.

[3] S. Dimopoulos, S. Thomas and J.D. Wells, Nucl. Phys. 488 (1997) 39.

[4] H. Pagels, J.R. Primack, Phys. Rev. Lett. 48 (1982) 223.

[5] T. Moroi, H. Murayama, M. Yamaguchi, Phys. Lett. B303 (1993) 289.

[6] A. Gouvêa, T. Moroi, H. Murayama, Phys. Rev. D56 (1997) 1281.

[7] R. Kallosh, L. Kofman, A. Linde, A.V. Proeyen, Phys. Rev. D61 (2000) 103503; G.F. Giudice, A. Riotto, I. Tkachev, JHEP 9908 (1999) 009.

[8] M.Y. Khlopov, A.D. Linde, Phys. Lett. B138 (1984) 265; J. Ellis, J.E. Kim, D.V. Nanopoulos, Phys. Lett. B145 (1984) 181; M. Kawasaki, T. Moroi, Prog. Theor. Phys. 93 (1995) 879.

[9] S. Dimopoulos, R. Esmailzadeh, L.J. Hall, G.D. Starkman, Astrophys. J. 330, 545 (1988); Nucl. Phys. B311, 699 (1989).

[10] M.H. Reno, D. Seckel, Phys. Rev. D37 (1988) 3441.

[11] M. Bolz, W. Buchmüller, M. Plümacher, Phys. Lett. B443 (1998) 209.

[12] E. Holtmann, M. Kawasaki, K. Kohri, T. Moroi, Phys. Rev. D60 (1999) 023506.

[13] T. Ghergetta, G.F. Giudice, A. Riotto, Phys. Lett. B446 (1999) 28.

[14] K. Griest, D. Seckel, Phys. Rev. D43 (1991) 3191.

[15] J. Gratsias, R.J. Scherrer, D.N. Spergel, Phys. Lett. B262 (1991) 298; M. Kawasaki, T. Moroi, Phys. Lett. B346 (1995) 27.

[16] M. Fukugita, T. Yanagida, Phys. Lett. B174 (1986) 45.

[17] See, for recent reviews, W. Buchmüler, M. Plümacher, Phys. Rep. 320 (1999) 329; M. Plümacher, Nucl. Phys. B530 (1998) 207 and references there in.

[18] K. Kumekawa, T. Moroi, T. Yanagida, Prog. Theor. Phys. 92 (1994) 437; G. Lazarides, hep-ph/9904428 and reference therein; G.F. Giudice, M. Peloso, A. Riotto, I. Tkachev, JHEP 9908 (1999) 014; T. Asaka, K. Hamaguchi, M. Kawasaki, T. Yanagida, Phys. Lett. B464 (1999) 12; Phys. Rev. D61 (2000) 083512.

[19] D.H. Lyth, E.D. Stewart, Phys. Rev. Lett. 75 (1995) 201; Phys. Rev. D53 (1996) 1784. 